\title{
Zeitprofilkontrollen sind kein Grund zur Panik
}

_ Eine Mitteilung wegen einer Überschreitung der EBM-Zeitprofile ist selten und bedeutet auch nicht automatisch eine Honorarkürzung. Der Hintergrund ist ein ganz einfacher: Im Anhang 3 des EBM sind für die meisten ärztlichen Leistungen Prüfzeiten festgelegt. Dies ist so geschehen, um die Bewertungen der Leistungen begründen zu können. Die ärztliche Arbeitszeit wurde kalkuliert und der Bewertung zugrunde gelegt.

Ärgerlich und unlogisch ist dabei natürlich, dass eine derart sauber hinterlegte ärztliche Tätigkeit dann im Rahmen von Begrenzungsmaßnahmen wie dem Regelleistungsvolumen (RLV) nur budgetiert vergütet wird. Nach höchst- richterlicher Entscheidung ist das aber kein Grund, im Gegenzug eine geringere Prüfzeit einzufordern.

\section{MMW-KOMMENTAR}

Klar ist deshalb in erster Linie, dass für eine Leistung im EBM die vorgegebene Mindestzeit eingehalten werden muss, z. B. die 15 Minuten bei den psychosomatischen Leistungen nach den Nrn 35100 und 35110 EBM. Wer Zeitobergrenzen - 12 Stunden an einzelnen Tagen an mehr als drei Tagen in einem Quartal oder 780 Stunden in einem Quartal - verletzt, muss sich aber erst einmal keine Sorgen machen: Sie sind nur "Aufgreifkriterien" für eine Plausibilitätsprüfung. Wird ein Arzt an dieser Stelle auffällig, fordert die Prüfstelle

\section{Wann Verbände nach GOÄ berechnet werden können}

_ Nach den Allgemeinen Bestimmungen des Kapitels C I der GOÄ sind Wundverbände nach Nr. 200 im Zusammenhang mit einer operativen Leistung, Punktionen, Infusionen, Transfusionen oder Injektionen nicht gesondert berechnungsfähig. Der Begriff „operative Leistung " würde dabei eigentlich bedeuten, dass der Verband durch eine Operationswunde notwendig wird. Wundversorgungen nach den Nrn. 2000-2006 wären also nicht betroffen - eigentlich! Der GOÄ-Ausschuss der Bundesärztekammer ist jedoch zu dem Ergebnis gekommen, dass die Nr. 200 nur neben der Nr. 2006 abrechenbar ist. Bei den anderen Nrn. wird nämlich in den Legenden auf „Naht“ und/oder „Umschneidung“ abgestellt. Zudem beinhalten die Leistungen nach den Nrn. 2000 und 2003 be- reits den Verband, was eine gesonderte Abrechnung in jedem Fall ausschließt.

\section{MMW-KOMMENTAR}

Der Ausschluss gilt allerdings nur für den einfachen Verband, nicht für Funktionsverbände. Deshalb sind z.B. die Verbände nach den Nrn. 204 oder 208 auch neben den Wundversorgungs-Nrn. 2000-2005 berechnungsfähig. Auch der Zuschlag nach Nr. 208 könnte isoliert berechnet werden, wenn er im Rahmen eines Verbands anfallen würde, der nach Nr. 200 berechnet werden könnte. Werden im Rahmen einer Wundbehandlung Externa aufgetragen, kommt die Nr. 209 GOÄ zum Ansatz.

Insbesondere bei der Behandlung einer sekundär heilenden Wunde wie z. B. einem Ulcus cruris ergeben sich deshalb sehr weitgehende Abrechnungsmöglichkeiten. Nicht zunächst nur eine Stellungnahme an. Wurde die Quartalsobergrenze überschritten, sollte man die abgerechneten Einzelleistungen und Quartalspauschalen prüfen. Bei dreimaliger Überschreitung der Tagesobergrenze sollte man zusätzlich prüfen, ob Leistungen mit festen Zeitvorgaben der Grund waren. Ist das der Fall, und wurden diese Leistungen tatsächlich innerhalb der jeweiligen Zeitvorgaben erbracht, genügt der Hinweis, dass an den betreffenden Tagen tatsächlich Arbeitszeiten in dieser Größenordnung angefallen sind. Bei der Plausibilitätsprüfung kommt es nämlich nicht zu einer Beweisumkehr, wie man sie etwa aus der Wirtschaftlichkeitsprüfung bei einem „offensichtlichen Missverhältnis" kennt.

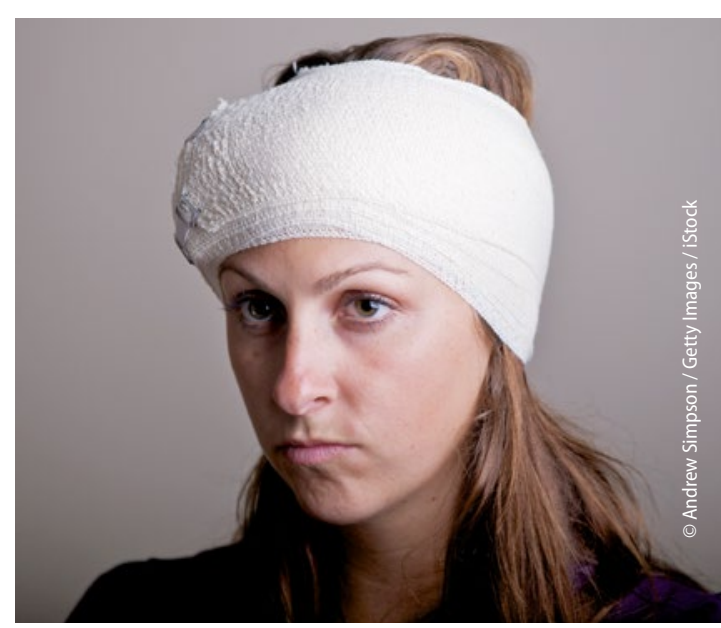

Zirkulärer Kopfverband (Nr. 204 GOÄ): neben Wundversorgung abrechenbar.

vergessen sollte man auch, dass die im Zusammenhang mit den ambulant erbrachten Leistungen des Abschnitts CI. angefallenen Kosten für Verbrauchsmaterialien gemäß § 10 GOÄ berechnet werden können und zwar auch für Verbände, die unter eine Ausschlussregelung fallen. 